\title{
Rate of convergence for the Euclidean minimum spanning tree limit law
}

\author{
Patrick Jaillet \\ MSIS Department, CBA 5.202, University of Texas at Austin, Austin, TX, USA
}

Received October 1992

Revised June 1993

Let $N_{n}$ be the number of points of a Poisson point process of intensity $n$ times the Lebesgue measure over $[0,1]^{2}$, and let $L_{\mathrm{MST}}\left(N_{n}\right)$ be the length of the optimal spanning tree connecting these $N_{n}$ points. It is well-known that there is a constant $0<\beta_{\mathrm{MST}}<\infty$ such that $\lim _{n \rightarrow \infty} \mathrm{E} L_{\mathrm{MST}}\left(N_{n}\right) / \sqrt{n}=\beta_{M S T}$. In this paper we give the exact rate of convergence for this limiting behavior.

Euclidean minimum spanning tree; asymptotic analysis; rate of convergence

\section{Introduction}

The minimum spanning tree problem (MSTP) in the plane requires finding the length of the shortest tree spanning $n$ points of $\mathbf{R}^{2}$. We are concerned here with stochastic versions of the problem. First let $X_{i}, 1 \leq i<\infty$, be uniformly and independently distributed random variables in $[0,1]^{2}$ and let $L_{\mathrm{MST}}(n)$ be the length of the shortest tree spanning $\left\{X_{1}, X_{2}, \ldots, X_{n}\right\}$. Steele [9] proved that $L_{\mathrm{MST}}(n)$ is asymptotic to $\beta_{\mathrm{MST}} \sqrt{n}$ with probability one (the same being true in expectation). In fact this result is valid for any uniform i.i.d. random variables with compact support of measure one in $\mathbf{R}^{d}, d \geq 2$, provided $\sqrt{n}$ is replaced by $n^{(d-1) / d}$, the constant depending only on the dimension of the space and not on the shape of the compact support. Similar results had previously been obtained for the traveling salesman problem, the weighted matching problem, and the Steiner tree problem (Beardwood et al.

* This research was supported in part by a joint CNRS/NSF Grant.

Correspondence to: P. Jaillet, MSIS Department, CBA 5.202, The University of Texas at Austin, Austin, Texas 78712, USA, and, Laboratoire de Mathématiques Appliquées, Ecole Nationale des Ponts et Chaussées, 93167 Noisy-le-Grand, France.
[2] and Steele [8]). Questions about rates of convergence for these limit laws have been raised many times in the literature (see for example $[2,5,8,9])$. There are in fact two issues concerning information on rates of convergence (let $P$ be a generic symbol representing any of the problems pre-cited):

1. What is the asymptotic size of $L_{P}(n)-$ $\mathbf{E} L_{P}(n)$ ?

2. What can be said about the rate of convergence of the normalized means $\mathbf{E} L_{P}(n) / \sqrt{n}$ to $\beta_{P}$ ?

With respect to the first question, Rhee and Talagrand [7] proved that, for the TSP, there is a constant $k$ such that $\left\|L_{\mathrm{TSP}}(n)-\mathbf{E} L_{\mathrm{TSP}}(n)\right\|_{p} \leq$ $k \sqrt{p}$ for each $p$ for all $n$. This interesting result indicates that $L_{\mathrm{TSP}}(n)$ is quite concentrated around its mean. With respect to the second question, some partial results have been obtained in Jaillet [4], where it is proved that $\left|\mathbf{E} L_{P}(n)\right|$ $\sqrt{n}-\beta_{P} \mid=\mathrm{O}(1 / \sqrt{n})$ (see also Alexander [1]). However, the important question remained open: Is $1 / \sqrt{n}$ the exact rate of convergence, or does $\mathrm{E} L_{P}(n) / \sqrt{n}$ go faster to $\beta_{P}$ ?

In this paper, we consider the case of the MST and give an answer to this question for a Poisson point process $\pi_{n}$ of intensity $n$ times the Lebesgue measure over $[0,1]^{2}$. More precisely, let $N_{n}$ be a 
Poisson random variable with parameter $n$ representing the number of points of this process in $[0,1]^{2}$. We prove that $\left|\mathbf{E} L_{\mathrm{MST}}\left(N_{n}\right) / \sqrt{n}-\boldsymbol{\beta}_{\mathrm{MST}}\right|$ $=\Theta(1 / \sqrt{n})$.

The paper is organized as follows. In Section 2, we state, in the context of a Poisson point process, some simple results of [4]. In Section 3, we prove our main result. Finally in Section 4, we give some final remarks.

\section{Background results}

If one follows the usual subadditivity argument (such as in $[2,8]$ ), one can go one step further and show that $\mathrm{E} L_{\mathrm{MST}}\left(N_{n}\right) \geq \beta_{\mathrm{MST}} \sqrt{n}-k_{1}$ for a positive constant $k_{1}$. Also, adapting a classical argument given in [2] for the TSP, one can show that $\mathrm{E} L_{\mathrm{MST}}\left(N_{n}\right) \leq \beta_{\mathrm{MST}} \sqrt{n}+k_{2}$ for a positive constant $k_{2}$. Let us present these arguments (adapted from [4]), and combine the results in one lemma.

Lemma 1. Let $N_{n}$ be the number of points of a Poisson point process $\pi_{n}$ of intensity $n$ times the Lebesgue measure over $[0,1]^{2}$, and let $L_{\mathrm{MST}}\left(N_{n}\right)$ be the length of the minimum spanning tree connecting these $N_{n}$ points. Then we have

$\left|\mathbf{E} L_{\mathrm{MST}}\left(N_{n}\right) / \sqrt{n}-\beta_{\mathrm{MST}}\right|=0(1 / \sqrt{n})$.

Proof Let us first prove that there exists a constant $k_{1}$ such that

$\mathbf{E} L_{\mathrm{MST}}\left(N_{n}\right) \geq \beta_{\mathrm{MST}} \sqrt{n}-k_{1}$.

Consider $x=\left\{x_{i}: 1 \leq i<\infty\right\}$ to be an arbitrary infinite sequence of points in $[0,1]^{2}$, and let $x^{(n)}$ $=\left\{x_{1}, x_{2}, \ldots, x_{n}\right\}$ be its first $n$ points. Let $\left\{Q_{i}\right.$ : $\left.1 \leq i \leq m^{2}\right\}$ be a partition of $[0,1]^{2}$ into $m^{2}$ squares with edges parallel to the axes and of side length $1 / m$. Then there exists a constant $k_{1}$ such that

$L_{\mathrm{MST}}\left(x^{(n)}\right) \leq \sum_{i=1}^{m^{2}} L_{\mathrm{MST}}\left(x^{(n)} \cap Q_{i}\right)+k_{1} m$.

The argument, classical, has its origin in [2, Lemma 1] and has been used subsequently in many papers. Consider the following tree construction connecting $x^{(n)}$ (see Figure 1 for an illustration): first construct optimal trees connecting $x^{(n)} \cap Q_{i}$ for $1 \leq i \leq m^{2}$. Then, in each square $Q_{i}$ where $x^{(n)} \cap Q^{i}$ is not empty, choose one point as a representative and finally construct an

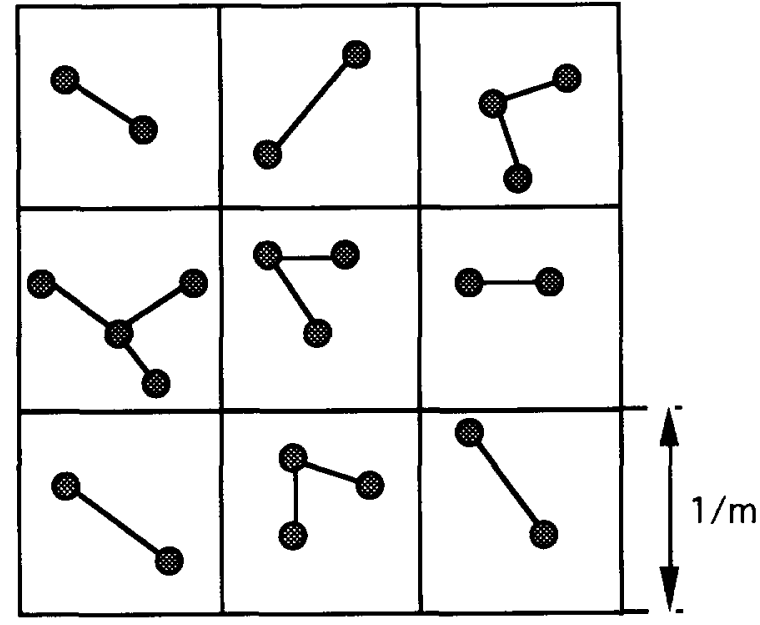

(i) Find MST trees in the subsquares

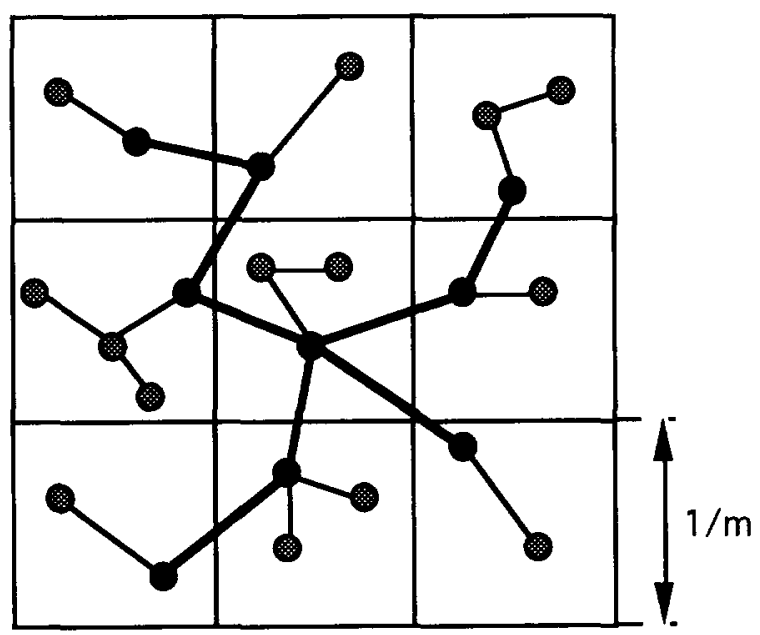

(ii) Patch the MST trees together by connecting their representatives

Fig. 1. Tree construction on $x^{(n)}$ in $[0,1]^{2}$

optimal tree connecting the set $S$ of all representatives (at most $m^{2}$ points). The combination of the small trees together with the large tree gives a spanning tree connecting $x^{(n)}$ of length $\sum_{i=1}^{m^{2}}$ $L_{\mathrm{MST}}\left(x^{(n)} \cap Q_{i}\right)+L_{\mathrm{MST}}(S)$. Now it is easy to show (see [3]) that there exists a constant $k_{1}$ such that $L_{\mathrm{MST}}(S) \leq k_{1} \sqrt{|S|}$, and this establishes (3). From (3) we then have, starting with a Poisson point process $\pi_{m^{2} n}$ on $[0,1]^{2}$ and using an obvious scaling property, the following subadditive inequality:

$\mathrm{E} L_{\mathrm{MST}}\left(N_{m^{2} n}\right) \leq m \mathbf{E} L_{\mathrm{MST}}\left(N_{n}\right)+k_{1} m$. 
Dividing both sides of (4) by $\sqrt{m^{2} n}$, letting $m$ go to infinity, and using the fact that $\mathbf{E} L_{\mathrm{MST}}\left(N_{m^{2} n}\right) /$ $\sqrt{m^{2} n}$ goes to $\beta_{\text {MST }}$ when $m$ goes to infinity, we finally obtain (2). Now let us prove that there exists a constant $k_{2}$ such that

$\mathrm{E} L_{\mathrm{MST}}\left(N_{n}\right) \leq \beta_{\mathrm{MST}} \sqrt{n}+k_{2}$.

From the arguments given above, it will be enough to prove that there exists a constant $k_{2}$ such that

$L_{\mathrm{MST}}\left(x^{(n)}\right) \geq \sum_{i=1}^{m^{2}} L_{\mathrm{MST}}\left(x^{(n)} \cap Q_{i}\right)-k_{2} m$.

Indeed, one would then have the following inequality:

$\mathbf{E} L_{\mathrm{MST}}\left(N_{m^{2} n}\right) \geq m \mathbf{E} L_{\mathrm{MST}}\left(N_{n}\right)-k_{2} m$,

which can be analyzed in the same manner as inequality (4). The argument for proving (6) is also classical and is adapted from [2, Lemma 2] (see Figure 2 for an illustration). Let $T^{*}$ be an optimal tree through $x^{(n)}$ and let us suppose that $x^{(n)} \cap Q_{i}$ is not empty. Let $T_{i}^{*}=T^{*} \cap Q_{i}$ and let $T_{i j}$ for $1 \leq j \leq \mu_{i}\left(\mu_{i} \leq\left|x^{(n)} \cap Q_{i}\right|\right)$ be the connected pieces of $T_{i}^{*}$ which contain at least an element of $x^{(n)}$. Let $l_{i}$ be the total length of all these connected pieces. By using some portion of the perimeter of $Q_{i}$ one can connect endpoints of these pieces (lying on the perimeter of $Q_{i}$ ) in order to form a tree spanning $x^{(n)} \cap Q_{i}$. Now, the additional points, used for this connection, lie outside of the convex hull of $x^{(n)} \cap Q_{i}$. Hence we have $L_{\mathrm{MST}}\left(x^{(n)} \cap Q_{i}\right) \leq l_{i}+\operatorname{per}\left(Q_{i}\right)=l_{i}+4 / m$. By summing both sides for all $i$, we get the validity of (6), with $k_{2}=4$.

\section{The main result}

The main result of this paper is the following theorem:

Theorem 1. Let $N_{n}$ be the number of points of a Poisson point process $\pi_{n}$ of intensity $n$ times the Lebesgue measure over $[0,1]^{2}$, and let $L_{\mathrm{MST}}\left(N_{n}\right)$ be the length of the optimal spanning tree connecting these $N_{n}$ points. Then

$$
\left|\mathbf{E} L_{\mathrm{MST}}\left(N_{n}\right) / \sqrt{n}-\beta_{\mathrm{MST}}\right|=\Theta(1 / \sqrt{n}) .
$$

Proof. From Lemma 1, it suffices to prove that there exists a positive constant $c$ such that

$\mathrm{E} L_{\mathrm{MST}}\left(N_{n}\right) / \sqrt{n} \geq \beta_{\mathrm{MST}}+c / \sqrt{n}$.

Let us first replace the traditional partitioning and patching way of getting the subadditivity inequality (as in Figure 1) by a recursive way. We divide $[0,1]^{2}$ into four squares with edges parallel to the axes and of side length $\frac{1}{2}$ and we solve the MSTP in each of them. Then we select in each (not empty) quadrant the point closest to the

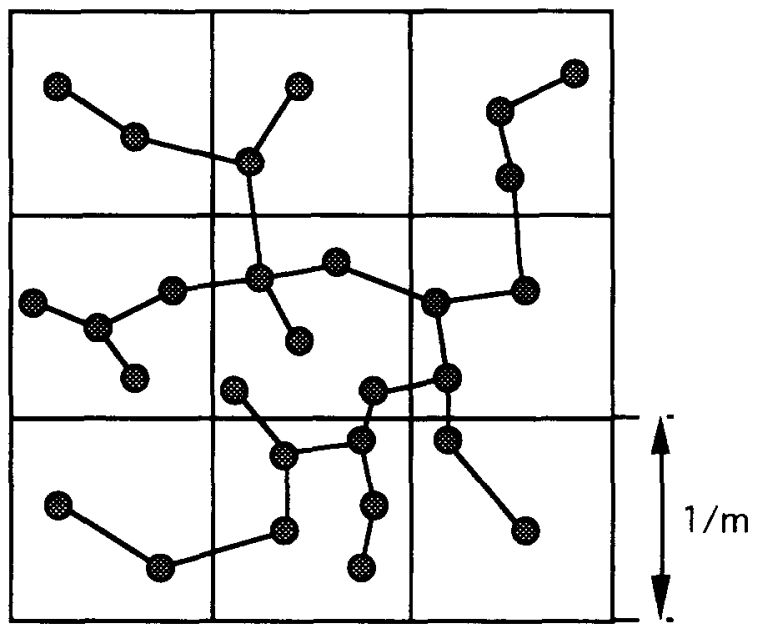

(i) Find a MST tree spanning all points

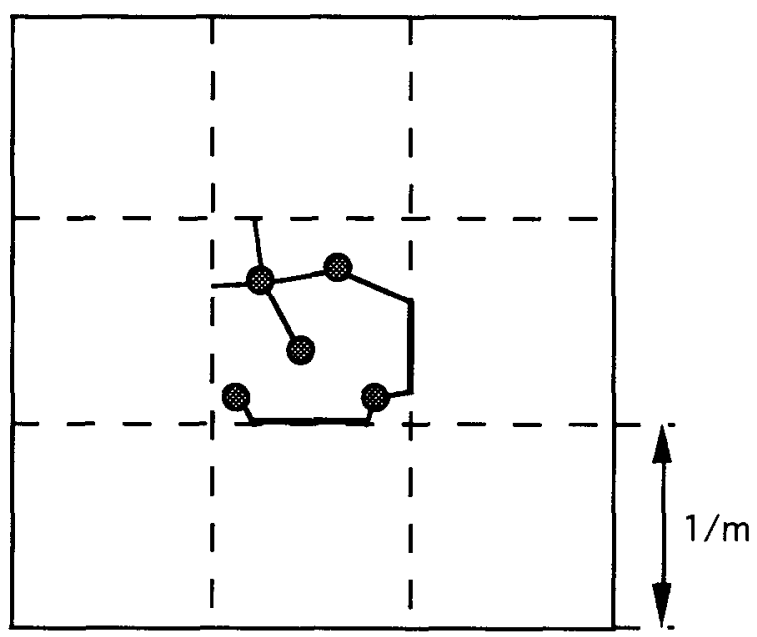

(ii) Connect the components in a subsquare, using part of the perimeter

Fig. 2. Tree construction on $x^{(n)} \cap Q_{i}$ in $[0,1]^{2}$ 
center of $[0,1]^{2}$, and finally we construct a tree connecting these points (see Figure 3, parts (i) and (ii)). Starting with the Poisson point process $\pi_{4 n}$ in $[0,1]^{2}$, we obtain

$\mathbf{E} L_{\mathrm{MST}}\left(N_{4 n}\right) \leq 2 \mathbf{E} L_{\mathrm{MST}}\left(N_{n}\right)+k / \sqrt{n}$,

where $k$ is a positive constant. By using this inequality recursively we get

$$
\begin{aligned}
& \mathbf{E} L_{\mathrm{MST}}\left(N_{4^{m} n}\right) \\
& \quad \leq 2^{m} \mathbf{E} L_{\mathrm{MST}}\left(N_{n}\right)+2^{m-1}\left(1-\left(\frac{1}{4}\right)^{m}\right) 4 k / 3 \sqrt{n} .
\end{aligned}
$$

Dividing each side by $\sqrt{4^{m} n}$, and letting $m$ go to infinity, we get

$$
\frac{\mathbf{E} L_{\mathrm{MST}}\left(N_{n}\right)}{\sqrt{n}} \geq \beta_{\mathrm{MST}}-\frac{2 k}{3 n},
$$

which is already an improvement on (2). Can we do better? Yes. The main idea is to improve the feasible solution, obtained from the connection of the 4 trees, by considering potential savings along the borderline of two given adjacent subsquares. Figure 3 part (iii) illustrates such savings. Note that there will be savings each time there exists a point in one of the subsquares which has its closest point (among $\pi_{4 n}$ ) that is located in an another subsquare. In order to evaluate the size and likelihood of these savings, let us refer to Figure 4, where we show two concentrated balls centered on the borderline of two subsquares, of radius $r$ and $4 r$, respectively. Now consider the following event $\mathscr{H}$ :

There is exactly one point in region $A$, no point in region $B$, and at least one point in region $C$.

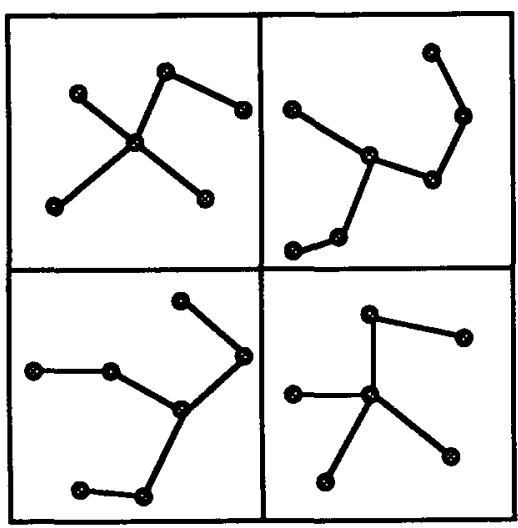

(i) The four optimal trees

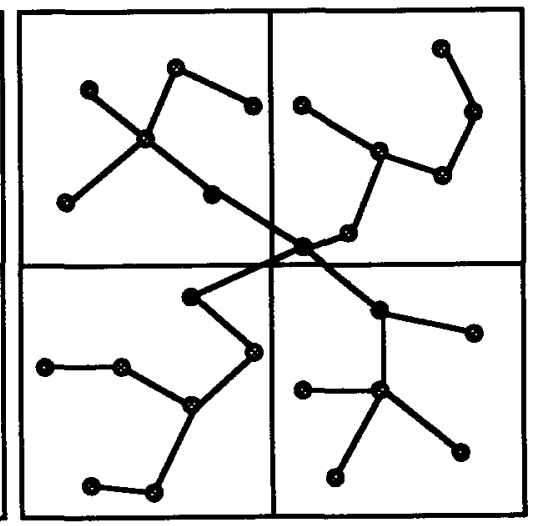

(ii) Connection of the four optimal trees

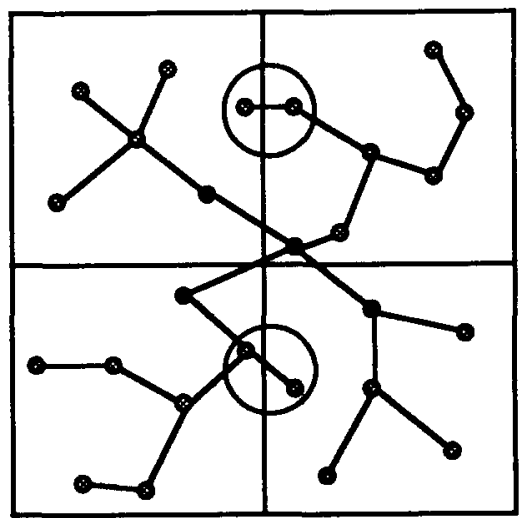

(iii) The circles indicate areas where savings have been possible.

Fig. 3. Construction of a feasible solution and 'post-savings' 


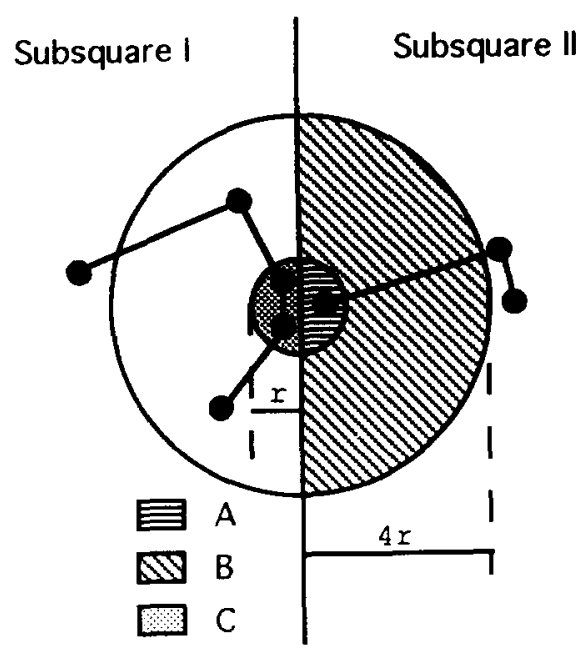

(i) Two concentric squares centered on the border of two subsquares

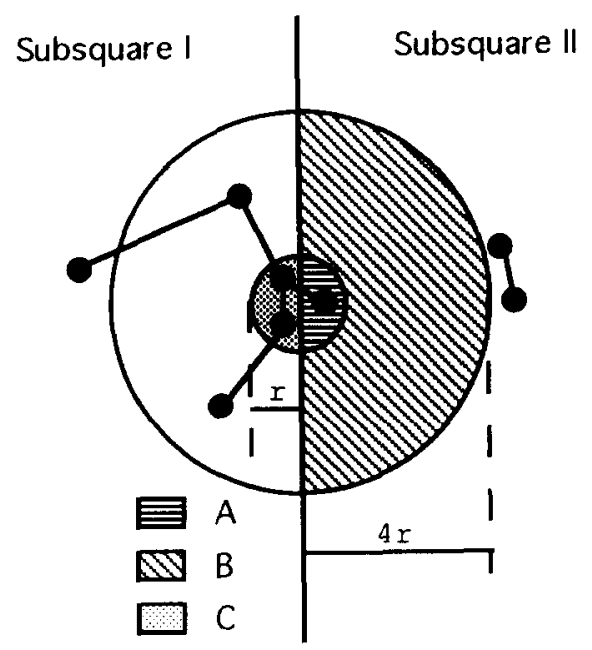

(ii) Exchange of two edges with savings of at least $r$

Fig. 4. Conditions for savings

If such an event is true, then, by connecting the point of $A$ to one of the points of $C$, one gets savings of at least $3 r-2 r=r$ (see Figure 4 (ii)). What is the probability of this event when one considers a Poisson point process $\pi_{4 n}$ in $[0,1]^{2}$ ? For $r=\alpha / \sqrt{n}$, where $\alpha$ is any positive constant, one can always find $n$ large enough, so that this probability is greater than or equal to a positive constant, say $a$ (indeed, if $r=\alpha / \sqrt{n}$, the number of points in region $A, B$, and $C$ are all given by independent Poisson random variables with constant parameters). Along the side of two adjacent squares (of length $\frac{1}{2}$ ), one can pack at least $\lfloor\sqrt{n} /(8 \alpha)\rfloor$ non-overlapping, and thus independent, such combination of two concentric balls. The expected total savings will then be bounded from below by

$a(\alpha / \sqrt{n})(\lfloor\sqrt{n} /(8 \alpha)\rfloor) \geq c_{1}$,

where $c_{1}$ is a positive constant. Instead of (10), we now have

$\mathbf{E} L_{\mathrm{MST}}\left(N_{4 n}\right) \leq 2 \mathbf{E} L_{\mathrm{MST}}\left(N_{n}\right)+k / \sqrt{n}-c_{1}$.

This in turn implies that, for any positive constant $c<c_{1}$, there exists $n(c)$ large enough such that for all $n \geq n(c)$,

$$
\mathbf{E} L_{\mathrm{MST}}\left(N_{4 n}\right) \leq 2 \mathbf{E} L_{\mathrm{MST}}\left(N_{n}\right)-c \text {. }
$$

By using this inequality recursively we get

$$
\mathbf{E} L_{\mathrm{MST}}\left(N_{4^{m} n}\right) \leq 2^{m} \mathbf{E} L_{\mathrm{MST}}\left(N_{n}\right)-c\left(2^{m}-1\right) \text {. }
$$

Dividing each side by $\sqrt{4^{m} n}$, and letting $m$ go to infinity, we finally get the desired result.

\section{Concluding remarks}

The techniques and results developed in this paper remain valid for other functionals of geometric probability. For the Steiner tree problem and the minimum weight matching problem, the arguments are in fact almost identical. However, for the traveling salesman problem, difficulties may arise. Indeed, going back to Figure 4 (i), and assuming that event $\mathscr{H}$ is true, then, if one decides to visit the unique point of region $A$ by a double link from one of the points of region $C$, savings will not necessarily occur with certainty (the point in $A$ could indeed be very close to the line joining its predecessor and successor along the previous TSP tour). In a first version of this paper, we conjectured that these savings would occur with a constant probability, still implying a rate of $\Theta(1 / \sqrt{n})$ for the TSP. In fact, Rhee [6] has recently and independently proved this result. The basic idea of her proof is identical to ours, although it involves solving a number of significant technical problems in order to insure that 
such savings indeed occur with a constant probability.

Finally, for all these problems, it is natural to expect that $\left|\mathbf{E} L_{P}(n) / \sqrt{n}-\beta_{P}\right|=\Theta(1 / \sqrt{n})$ remains true under the uniform fixed sample sized model. However, as pointed out in [6], this does not seem to be an easy consequence of the corresponding result stated under the Poisson model. The usual way to link the two models (see [4] for details) is to prove that $\left|\mathbf{E} L_{P}(k+1)-\mathbf{E} L_{P}(k)\right|$ $=O(1 / \sqrt{k})$, which then implies that $\mid \mathbf{E} L_{P}\left(N_{n}\right)$ - $\mathbf{E} L_{P}(n) \mid=O(1)$. This last relationship is however not sufficient here and a deeper understanding of how $\left|\mathbf{E} L_{P}(k+m)-\mathbf{E} L_{P}(k)\right|$ behaves as a function of $m$ and $k$ seems to be necessary for closing the gap.

\section{Acknowledgements}

The author would like to thank an anonymous referee for bringing to his attention the papers by Alexander [1] and Rhee [6].

\section{References}

[1] K. Alexander, "A note on some rates of convergence in first-passage percolation", Department of Mathematics, University of Southern California, Los Angeles, 1992.

[2] J. Beardwood, J. Halton and J. Hammersley, "The shortest path through many points", Proc. Camb. Phil. Soc. 55, 299-327 (1959).

[3] E. Gilbert, "Random minimal trees", J. SLAM 13, 376-387 (1965).

[4] P. Jaillet, "Rates of convergence for quasi-additive smooth Euclidean functionals and application to combinatorial optimization problems", Math. Oper. Res. 17, 965-980 (1992).

[5] R. Karp, "Probabilistic analysis of partitioning algorithms for the traveling salesman problem in the plane", Math. Oper. Res. 2, 209-224 (1977).

[6] W. Rhee, "Boundary effects in the travelling salesperson problem", Department of Management Science, The Ohio State University, Columbus, 1992.

[7] W. Rhee and M. Talagrand, "A sharp deviation inequality for the stochastic traveling salesman problem", Ann. Prob. 17, 1-8 (1988).

[8] J. Steele, "Subadditive Euclidean functionals and nonlinear growth in geometric probability", Ann. Prob. 9, 365376 (1981).

[9] J. Steele, "Growth rates of Euclidean minimal spanning trees with power weighted edges", Ann. Prob. 16, 17671787 (1988). 